\title{
Influence of pupil size on measurements made with the Lens Opacity Meter 701
}

\author{
Michael P Clarke, James C G Pearson, Stephen A Vernon, J Colin Matthews
}

\begin{abstract}
The Lens Opacity Meter 701 (Interzeag, Switzerland) is a recently developed instrument for assessing cataract. It measures the degree of scatter of a red light beam by the lens. Fifty patients prior to cataract surgery had measurements made before and after dilatation of the pupil. Inconsistent results were found at pupil diameters of less than $4 \mathrm{~mm}$. It is postulated that this was due to absorption of scattered light by the iris.
\end{abstract}

The lens opacity meter 701 (LOM) (Interzeag, Switzerland) measures the back scattering of a $1.5 \mathrm{~mm}$ beam of $700 \mathrm{~nm}$ wavelength directed along the visual axis. The scattered light is detected by a photocell $27^{\circ}$ below the incident beam. The instrument provides a quick, reproducible, numerical measurement which correlates well with slit-lamp grading. ${ }^{12}$ Although it is possible to make consistent measurements through an undilated pupil, the manufacturers recommend that the pupil diameter should be at least $4 \mathrm{~mm} .^{1}$ This limits the use of the instrument in patients with posterior synechiae and those on chronic miotic therapy. Normal values of lens scatter adjusted for age have been calculated with the Lens Opacity Meter 701. ${ }^{2}$ Tuft et $a l^{3}$ found a significant relationship between Lens Opacity Meter reading and Snellen acuity and age, but concluded that the LOM was more suited to the evaluation of the contribution of lens opacities to visual loss in populations rather than individuals.

We have studied lens scatter in patients with cataract prior to surgery and have assessed the influence of pupil size on the readings.

Medical Centre,

Nottingham

M P Clarke

S A Vernon

\section{Department of}

Epidemiology and

Community Medicine,

Queen's Medical Centre,

Nottingham

J C G Pearson

Department of Physics, University of Nottingham J C Matthews

Correspondence to:

M P Clarke, FRCS.

Accepted for publication 3 May 1990

Table 1 All data

\begin{tabular}{llllllllll}
\hline Pupil & Subsets & Values & $d f$ & Variance & $S D$ & $F$ & $p$ & \\
\hline$<4$ & 42 & 94 & 52 & 43.07 & 6.56 & & & & \\
$\geqslant 4$ & 50 & 125 & 75 & 19.79 & 4.45 & 2.17 & 12.97 & $<0.01$ & \\
$>4$ & 36 & 77 & 41 & 3.32 & 1.82 & & & & \\
\hline
\end{tabular}
Patients and methods consented to be examined on the LOM. The pupil size was reduced by increasing the background illumination. In a small number of cases this was supplemented by $1 \%$ pilocarpine. The pupils were then enlarged by dimming the background light. Tropicamide $1 \%$ was then instilled to dilate the pupils further. Sequential recordings were made of lens scatter at the pupil diameters obtained.
Table 2 Analysis of variance of lens scatter at pupil diameters of $<4 \mathrm{~mm}$ and $>4 \mathrm{~mm}$

\begin{tabular}{llllllll}
\hline Pupil & Subsets & Values & $d f$ & Variance & $S D$ & $F$ & $p$ \\
\hline$<4$ & 17 & 38 & 21 & 29.60 & 5.44 & & \\
$>4$ & 17 & 37 & 20 & 3.31 & 1.82 & & \\
\hline
\end{tabular}

Table 3 Analysis of variance of lens scatter at pupil diameters of $<4 \mathrm{~mm}$ and $\geqslant 4 \mathrm{~mm}$

\begin{tabular}{llllllll}
\hline Pupil & Subsets & Values & $d f$ & Variance & $S D$ & $F$ & $p$ \\
\hline$<4$ & 22 & 49 & 27 & 28.77 & 5.36 & & \\
$\geqslant 4$ & 22 & 54 & 32 & 4.34 & 2.08 & & \\
\hline
\end{tabular}

The pupil diameter was measured by a grating incorporated into the instrument optics.

Five measurements of lens scatter were made at each pupil diameter and a mean value and standard deviation calculated.

\section{Results}

The variation in the repeated readings of lens scatter was measured by calculating the variance (the square of the standard deviation) for each subject and pooling these variances for all subsets. The analysis was carried out for the subsets of the data defined by pupil sizes less than and greater than $4 \mathrm{~mm}$ respectively. Those results relating to exactly $4 \mathrm{~mm}$ pupil size were treated by defining the 'greater than $4 \mathrm{~mm}$ ' subset in two ways - 'strictly greater than $4 \mathrm{~mm}$ ', and ' $4 \mathrm{~mm}$ or more' to see what the effect of including those results on the boundary would be.

The first analysis used all of the available data, except where there was only one value in the subset, since this contributed no information on variation. Differences in variation were tested by the variance ratio $(F)$ test $^{4}$ (Table 1 ).

A problem with the above analysis was that

there was a lack of balance in the sets that were being compared, since some subjects contributed to one variance but not to the other, having multiple readings on only one side of the boundary. The analysis was therefore repeated, confined to those subjects who had two or more values on either side of the $4 \mathrm{~mm}$ division.

The first of these analyses looked at $<4 \mathrm{~mm}$ and $>4 \mathrm{~mm}$, with pupil size $4 \mathrm{~mm}$ excluded (Table 2). The analysis was then repeated with the upper group defined as $4 \mathrm{~mm}$ or more (Table 3).

\section{Discussion}

Clearly there is much greater variation in the readings when the pupil size is less than $4 \mathrm{~mm}$. The contrast between greater than $4 \mathrm{~mm}$ and 
Table 4 Percentage of illuminated cylinder detected at different pupil diameters and beam offsets

\begin{tabular}{|c|c|c|c|c|c|c|c|c|c|c|c|c|c|}
\hline \multirow[t]{2}{*}{ Pupil diam } & \multicolumn{13}{|c|}{ Offset (mm) } \\
\hline & $-1 \cdot 0$ & -0.9 & -0.8 & -0.7 & -0.6 & -0.5 & -0.4 & -0.3 & $-0 \cdot 2$ & $-0 \cdot 1$ & -0.0 & $0 \cdot 1$ & 0.2 \\
\hline $\begin{array}{l}2 \cdot 00 \\
2 \cdot 10 \\
2 \cdot 20 \\
2 \cdot 30 \\
2 \cdot 40 \\
2 \cdot 50 \\
2 \cdot 60 \\
2 \cdot 70 \\
2 \cdot 80 \\
2 \cdot 90 \\
3 \cdot 00 \\
3 \cdot 10 \\
3.20 \\
3 \cdot 30 \\
3 \cdot 40 \\
3 \cdot 50 \\
3 \cdot 60 \\
3 \cdot 70 \\
3 \cdot 80 \\
3 \cdot 90 \\
4 \cdot 00\end{array}$ & $\begin{array}{r}89 \\
91 \\
93 \\
94 \\
96 \\
96 \\
97 \\
98 \\
98 \\
99 \\
99 \\
99 \\
99 \\
99 \\
100 \\
100 \\
100 \\
100 \\
100 \\
100 \\
100\end{array}$ & $\begin{array}{c}91 \\
93 \\
95 \\
96 \\
97 \\
98 \\
98 \\
99 \\
99 \\
99 \\
99 \\
99 \\
99 \\
100 \\
100 \\
100 \\
100 \\
100 \\
100 \\
100 \\
100\end{array}$ & $\begin{array}{r}92 \\
94 \\
95 \\
96 \\
98 \\
98 \\
99 \\
99 \\
99 \\
99 \\
100 \\
100 \\
100 \\
100 \\
100 \\
100 \\
100 \\
100 \\
100 \\
100 \\
100\end{array}$ & $\begin{array}{r}92 \\
94 \\
96 \\
96 \\
97 \\
98 \\
98 \\
99 \\
99 \\
99 \\
99 \\
100 \\
100 \\
100 \\
100 \\
100 \\
100 \\
100 \\
100 \\
100 \\
100\end{array}$ & $\begin{array}{r}90 \\
93 \\
95 \\
95 \\
96 \\
97 \\
98 \\
99 \\
99 \\
99 \\
99 \\
99 \\
99 \\
100 \\
100 \\
100 \\
100 \\
100 \\
100 \\
100 \\
100\end{array}$ & $\begin{array}{r}88 \\
90 \\
92 \\
93 \\
95 \\
96 \\
97 \\
98 \\
98 \\
98 \\
99 \\
99 \\
99 \\
99 \\
99 \\
100 \\
100 \\
100 \\
100 \\
100 \\
100\end{array}$ & $\begin{array}{r}84 \\
86 \\
89 \\
91 \\
93 \\
94 \\
95 \\
96 \\
97 \\
98 \\
98 \\
98 \\
99 \\
99 \\
99 \\
99 \\
99 \\
99 \\
100 \\
100 \\
100\end{array}$ & $\begin{array}{r}78 \\
82 \\
85 \\
87 \\
89 \\
91 \\
93 \\
94 \\
95 \\
96 \\
97 \\
97 \\
98 \\
98 \\
99 \\
99 \\
99 \\
99 \\
99 \\
100 \\
100\end{array}$ & $\begin{array}{l}74 \\
76 \\
79 \\
82 \\
85 \\
87 \\
89 \\
91 \\
93 \\
94 \\
95 \\
96 \\
97 \\
97 \\
98 \\
99 \\
99 \\
99 \\
99 \\
99 \\
99\end{array}$ & $\begin{array}{l}68 \\
71 \\
74 \\
76 \\
80 \\
82 \\
84 \\
87 \\
90 \\
92 \\
93 \\
94 \\
95 \\
96 \\
97 \\
98 \\
98 \\
99 \\
99 \\
99 \\
99\end{array}$ & $\begin{array}{l}62 \\
65 \\
68 \\
71 \\
74 \\
77 \\
79 \\
83 \\
85 \\
87 \\
89 \\
91 \\
93 \\
94 \\
95 \\
96 \\
97 \\
98 \\
98 \\
98 \\
99\end{array}$ & $\begin{array}{l}56 \\
58 \\
61 \\
64 \\
68 \\
71 \\
75 \\
77 \\
80 \\
83 \\
85 \\
88 \\
89 \\
91 \\
93 \\
94 \\
95 \\
96 \\
97 \\
98 \\
98\end{array}$ & $\begin{array}{l}48 \\
52 \\
56 \\
59 \\
62 \\
65 \\
69 \\
71 \\
74 \\
78 \\
80 \\
83 \\
86 \\
88 \\
89 \\
91 \\
93 \\
94 \\
95 \\
96 \\
97\end{array}$ \\
\hline
\end{tabular}

$4 \mathrm{~mm}$ or more is less distinct. There is a large difference when the full data set is used. The difference is, however, much smaller when the matched sets are examined - variances 3.31 and $4 \cdot 34$ respectively $(F=1 \cdot 31, p>0 \cdot 5)$. This seems to suggest that those subjects where the range of pupil sizes was restricted had more variable results.

The most likely explanation for the lack of consistency in measurement of lens scatter when the pupil size is less than $4 \mathrm{~mm}$ is absorption of scattered light from the lens by the posterior surface of the iris. The photoreceptor for this scattered light lies at $27^{\circ}$ below the horizontal. On the assumption of a lens thickness of at least $3 \mathrm{~mm}$ it is possible to calculate the proportion of the scattered light from a beam of $1.5 \mathrm{~mm}$ in diameter which will be detected by a receptor at $27^{\circ}$ below the incident horizontal beam for different pupil diameters. If the whole of the illuminated cylinder is visible to the detector that is, is not masked by the iris - the signal will correspond to $100 \%$ of its 'correct' value. If the iris masks off half of the volume of the cylinder, then the signal will correspond to only $50 \%$ of its correct value - that is, the measured lens scatter will be underestimated by $50 \%$.

The beam is normally made to pass through the centre of the pupil. However, there may be advantage in deliberately raising it away from the centre of the iris, and such displacement will be called the offset of the beam. When the beam is raised, this will correspond to a negative offset.

Table 4 indicates the fraction of the illuminated cylinder seen by the photocell for a range of pupil diameters and a range of offsets. Although serial measurements on the same lens at pupil diameters less than $4 \mathrm{~mm}$ may be consistent at the same pupil diameter, these measurements will underestimate the 'true' lens scatter. This poses a difficulty if absolute thresholds of lens scatter measurement are used, as for example criteria for cataract surgery, or when serial measurements are made to assess changes in lens scatter with time. We conclude that the LOM is capable of providing a reproducible measurement of lens opacity provided the pupil diameter is $>4 \mathrm{~mm}$.

The authors thank the consultant ophthalmic surgeons at Queen's Medical Centre, Nottingham, for allowing their patients to take part in this study.

The authors have no proprietary, commercial, or financial interest in the Lens Opacity Meter 701.

1 Flammer J, Bebie H. Lens Opacity Meter: a new instrument to quantify lens opacity. Ophthalmologica 1987; 195: 69-72.

2 De Natale R, Flammer J, Zulauf M, Bebie T. Influence of age on the transparency of the lens in normals: a population study with the help of the Lens Opacity Meter 701. Ophthalmologica 1988; 196: 14-8.

3 Tuft SJ, Fitzke FW, Lawrenson J, Silver J, Marshall J. Quantification of lens opacification with a commercially available lensometer. Br F Ophthalmol 1990; 74: 78-81.

4 Kempthorne $O$. The design and analysis of experiments. New York: Wiley, 1960: 54-9. 